M. Arnaboldi et AL. 2004, ApJ, 614, L33

Preprint typeset using $\mathrm{LT}_{\mathrm{E}} \mathrm{X}$ style emulateapj v. 11/26/03

\title{
THE LINE-OF-SIGHT VELOCITY DISTRIBUTIONS OF INTRACLUSTER PLANETARY NEBULAE IN THE VIRGO CLUSTER CORE*
}

\author{
Magda Arnaboldi ${ }^{1}$, Ortwin Gerhard ${ }^{2}$, J. Alfonso L. Aguerri $^{3}$, Kenneth C. Freeman $^{4}$, \\ NicOla R. NAPOLITANO ${ }^{5}$, SADANORI OKAMURA ${ }^{6}$, NAOKI YASUDA $^{7}$ \\ ${ }^{1}$ INAF, Oss. Astr. di Torino, Strada Osservatorio 20, 10025 Pino Torinese, Italy. arnaboldi@to.astro.it \\ 2 Astronomical Institute, Univ. of Basel, Venusstrasse 7, CH-4102 Binningen, Switzerland. ortwin.gerhard@unibas.ch \\ ${ }^{3}$ Instituto de Astrofisica de Canarias, Via Lactea, 38200 La Laguna, Spain. jalfonso@iac.es \\ ${ }^{4}$ RSAA, Mt. Stromlo Observatory, Cotter Road, Weston Creek, ACT 2611, Australia. kcf@mso.anu.edu.au \\ ${ }^{5}$ Kapteyn Astronomical Institute, P.O.B. 800, 9700 AV Groningen, The Netherlands. nicola@astro.rug.nl \\ ${ }^{6}$ Dept. of Astronomy and RESCEU, School of Science, The Univ. of Tokyo, Tokyo 113-0033. okamura@astron.s.u-tokyo.ac.jp \\ ${ }^{7}$ Institute for Cosmic Ray Research, Univ. of Tokyo, Kashiwa, Chiba 277-8582. yasuda@icrr.u-tokyo.ac.jp \\ M. Arnaboldi et al. 2004, ApJ, 614, L33
}

\begin{abstract}
Radial velocities of 40 intracluster planetary nebulae (ICPNe) in the Virgo cluster were obtained with the new multi-fiber FLAMES spectrograph on UT2 at VLT. For the first time, the $\lambda 4959 \AA$ line of the [OIII] doublet is seen in a large fraction $(50 \%)$ of ICPNe spectra, and a large fraction of the photometric candidates with $\mathrm{m}(5007) \lesssim 27.2$ is spectroscopically confirmed.

ICPNe with the velocity dispersion of the Virgo cluster are found in our CORE field $1 .^{\circ} 2$ from M87. These may have originated from tidal mass loss of smaller galaxies in the M87 subcluster halo. In a field 0.25 deg from M87, we see an extended stellar halo of M87 in approximate dynamical equilibrium, but with few ICPNe. Finally, in a field near M84/M86, the ICPNe velocities are highly correlated with the galaxy velocities, showing that any well-mixed intracluster population is yet to form. Overall, the measured velocity distributions confirm the non-uniform dynamical structure and on-going assembly of the Virgo cluster.
\end{abstract}

Subject headings: (ISM:) planetary nebulae: general; galaxies: cluster: general; galaxies: cluster: individual (Virgo cluster); galaxies: evolution

\section{INTRODUCTION}

After the serendipitous discovery of intracluster planetary nebulae (ICPNe) in the Virgo (Arnaboldi et al. 1996) and Fornax (Theuns \& Warren 1997) clusters, our group embarked on a systematic study of the diffuse stellar population in the Virgo cluster. The aim of this research is to measure the fraction of cluster stars between galaxies, their radial distribution, and their motions. The discovery of ICPNe, the subsequent narrow band imaging surveys to find ICPN samples (Feldmeier et al. 1998, 2003a; Arnaboldi et al. 2002, 2003; Okamura et al. 2002), and the HST observations of Virgo cluster empty fields (Ferguson et al. 1998; Durrell et al. 2002) have revitalized the study of diffuse intracluster light (ICL). The preliminary results on the systematics of the ICL in different environments, from loose groups to rich clusters (Gonzalez et al. 2000, GalYam et al. 2003, Castro-Rodriguez et al. 2003, Feldmeier et al. $2003 \mathrm{~b}$ ), and the predictions from recent cosmological N-body and hydrodynamical simulations (Napolitano et al. 2003, Murante et al. 2004, Sommer-Larsen et al. 2004, Willman et al. 2004) have proven the study of the ICL as a valuable tool to investigate galaxy and galaxy cluster evolution.

ICPNe are the only component of the ICL whose kinematics can be measured at this time. This is important since the high-resolution N-body and hydrodynamical simulations predict that the ICL is unrelaxed, showing significant substructure in its spatial and velocity distributions in clusters similar to Virgo. While spatial structures have been observed in the ICPN number density distribution both in a single field (Okamura et al. 2002) and as field-to-field variations (Aguerri et al.

*BASED ON DATA COLLECTED WITH THE FLAMES SPECTROGRAPH AT THE UT2 OF THE VLT AT CERRO PARANAL, CHILE, OPERATED BY ESO, DURING OBSERVING RUN 71.B-0147(A)
2004), substructure in velocity space still needs to be investigated.

Early attempts by Freeman et al. (2000) with the AAT and $2 \mathrm{dF}$ spectrograph provided only a few spectroscopically confirmed emission line objects in the Virgo cluster core. Their identification as ICPNe relies on the presence of the weaker [OIII] line ( $\lambda$ 4959 $\AA)$ in the summed spectrum of all the detected single-line candidates. Because modern emission line candidate samples in Virgo contain a modest fraction of Ly $\alpha$ emitters at $z=3.1$ (Aguerri et al. 2004), spectral identification of ICPN either needs high spectral resolution to resolve the typical broad, asymmetric Ly $\alpha$ lines, and/or detection of the [OIII] $\lambda$ 4959/5007 $\AA$ doublet ${ }^{1}$.

ICPN samples are sparse, with only a few tens of ICPNe in a $0.25 \mathrm{deg}^{2}$ field, and their fluxes are faint, from $1 \times 10^{-16}$ to $5 \times 10^{-17} \mathrm{erg} \mathrm{cm}^{-2} \mathrm{~s}^{-1}$ in the [OIII] 5007 $\AA$ line. Spectroscopic observations thus require 8 meter class telescopes and spectrographs with a large field-of-view (FOV), of a fraction of a square degree. The [OIII] emission lines from PNe are only a few $\mathrm{km} \mathrm{s}^{-1}$ wide, so relatively high resolution spectra $(\mathrm{R}=7000$ to 10,000$)$ are desirable to reduce the sky contamination. The FLAMES-GIRAFFE spectrograph on VLT, with its medium-high spectral resolution, its FOV of 25 arcmin in diameter and 130 fibers in the MEDUSA mode, is therefore very well suited to this project. Here we present the results of the spectroscopic follow-up with FLAMES of the ICPN candidates selected from three survey fields in the Virgo cluster core.

${ }^{1}$ The emission line sample of Kudritzki et al. (2000) was even dominated by Ly $\alpha$ emitters; however, the luminosity function (LF) of this sample follows the LF of Ly $\alpha$ emitters in blank field surveys, not that of ICPNe in Virgo (Castro-Rodriguez et al. 2003). 
TABLE 1

OBSERVED FIELDS AND SPECTROSCOPIC CONFIRMATION RATES

\begin{tabular}{lccc}
\hline & FCJ $^{\mathrm{a}}$ & CORE $^{\mathrm{a}}$ & SUB $^{\mathrm{a}}$ \\
\hline$\alpha(\mathrm{J} 2000)$ & $12: 30: 47.6$ & $12: 27: 47.4$ & $12: 25: 33.8$ \\
$\delta(\mathrm{J} 2000)$ & $+12: 38: 32.4$ & $13: 21: 40.1$ & $+12: 47: 01.2$ \\
$m_{\text {lim }}(5007)^{\mathrm{b}}$ & 27.0 & 27.2 & 28.1 \\
$N_{\mathrm{Fib}}[<27.2]^{\mathrm{b}}$ & 18 & 34 & 18 \\
$N_{\mathrm{PN}}{ }^{\mathrm{b}}$ & 15 & 12 & 13 \\
$N_{\mathrm{PN}}(\lambda 4959)^{\mathrm{b}}$ & 10 & 5 & 4 \\
$N_{\mathrm{Fib}}\left(<m_{\min }\right)^{\mathrm{b}}$ & 13 & 34 & 18 \\
$N_{\mathrm{PN}}\left(<m_{\min }\right)^{\mathrm{b}}$ & 11 & 11 & 13 \\
Rate $\left(<m_{\min }\right)^{\mathrm{b}}$ & $84 \%$ & $32 \% \mathrm{c}$ & $72 \% \%^{\mathrm{d}}$ \\
${\text { Predicted Rate }\left(<m_{\min }\right)^{\mathrm{b}}}$ & $80 \%$ & $17 \%$ & $100 \% \mathrm{~d}$ \\
\hline
\end{tabular}

${ }^{a}$ References for photometric catalogues: Arnaboldi et al. (2002, FCJ), Aguerri et al. (2004, CORE), Okamura et al. (2002, SUB).

bymbols denote: limiting magnitude of photometric sample, $m_{\text {lim }}(5007)$; number of fibers allocated to objects with $m<27.2$ and not in bad CCD regions, $N_{\text {Fib }}[<27.2]$; number of detected PNe, $N_{\mathrm{PN}}$; number of detected PNe with double lines, $N_{\mathrm{PN}}(\lambda 4959)$; number of fibers allocated to objects brighter than $\min \left[27.2, m_{\text {lim }}(5007)\right], N_{\mathrm{Fib}}\left(<m_{\min }\right)$; number of detected such PNe, $N_{\mathrm{PN}}\left(<m_{m i n}\right) ;$ spectroscopic detection rate to $m_{\min }$, $\operatorname{Rate}\left(<m_{\min }\right)=N_{\mathrm{PN}}(<$ $\left.m_{\min }\right) / N_{\mathrm{Fib}}\left(<m_{\min }\right)$; corresponding rate predicted from photometry using simulations, Predicted Rate $\left(<m_{m i n}\right)=$ number of PNe in statistically decontaminated sample divided by total number of candidates in the field (Aguerri et al. 2004).

${ }^{\mathrm{c}}$ Higher than the predicted rate because in the fiber allocation priority was given to the brighter objects in the catalogue.

${ }^{\mathrm{d}}$ All candidates are true ICPNe, based on detections in both [OIII] and $\mathrm{H} \alpha$ narrow band images. Detected rate lower because of lower S/N.

\section{OBSERVATIONS}

Table 1 gives the three selected fields in the Virgo core, referred to as FCJ, CORE, and SUB (Aguerri et al. 2004). Spectra were acquired in service mode $(10 \mathrm{hrs}$ were allocated to this observing run, 71.B-0147, in priority A), with the FLAMES spectrograph at UT2 on VLT in the GIRAFFE+MEDUSA configuration. We used the low resolution grism LR 479.7, covering a wavelength range of $500 \AA$, centred on $4797 \AA$, and a spectral resolution of 7500 . This gives a velocity resolution of $40 \mathrm{~km} \mathrm{~s}^{-1}$, and a typical velocity error of $12 \mathrm{~km} \mathrm{~s}^{-1}$. The redshifted [OIII] emissions of ICPNe in the Virgo cluster core fall near the red edge of the grism response.

The FLAMES FOV covers the FCJ field entirely, and a significant fraction of both CORE and SUB. The total observing time was $2.5 \mathrm{hrs}$ for FCJ, and $2.6 \mathrm{hrs}$ each for CORE and SUB; the exposure times were based on the S/N estimate for detecting the [OIII] $5007 \AA$ line flux of $4.2 \times 10^{-17}$ erg $\mathrm{cm}^{-2} \mathrm{~s}^{-1}$, i.e. $\mathrm{m}(5007)=27.2$, with a $\mathrm{S} / \mathrm{N} \simeq 5$. Given that the [OIII] 4959/5007 $\AA$ emission lines have relative intensities 1:3, we expect to be able to detect the [OIII] 4959 $\AA$ emission for the brighter candidates only. The data reduction was carried out with the GIRAFFE pipeline, for CCD pre-reduction, fiber identification, wavelength calibration, geometric distortion corrections, co-addition and extraction of the final 1D-spectra.

Comparing with earlier measurements by Freeman et al. (2000), the RMS velocity difference for four ICPNe in common is $24 \mathrm{~km} \mathrm{~s}^{-1}$, where their velocity error was $40 \mathrm{~km} \mathrm{~s}^{-1}$.

\section{SPECTROSCOPIC RESULTS}

A total of 40 ICPN candidates were detected in the FLAMES spectra. In Fig. 1 we show single ICPN spectra

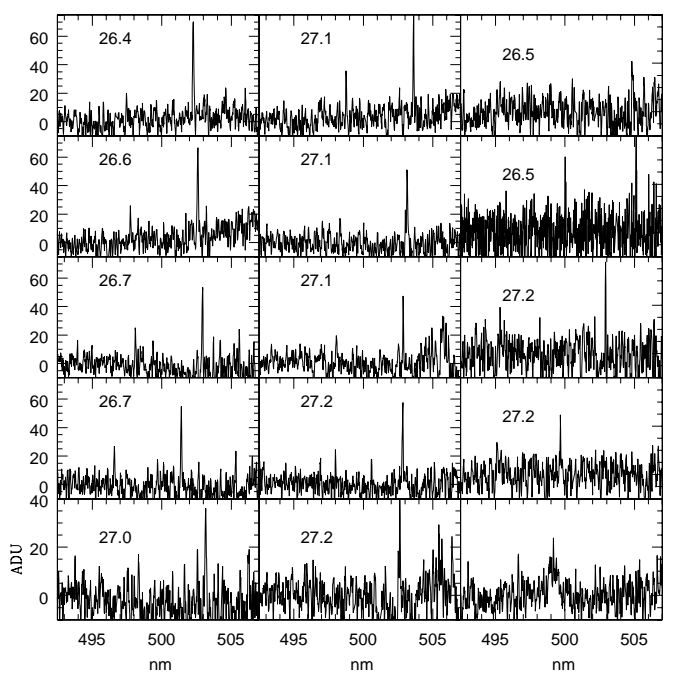

FIG. 1.- FLAMES spectra for 14 ICPNe observed in the FCJ (two left columns) and CORE fields. The spectrum in the lower right corner is a Ly $\alpha$ object, which shows a very broad line profile. The $m(5007)$ magnitudes are marked on the individual frames.

with the [OIII] 4959/5007 ̊̊ doublet, and the resolved spectrum of a $\operatorname{Ly} \alpha$ galaxy with a broad asymmetric line.

In Table 1 we give results for the three pointings individually. In the FCJ/CORE/SUB fields, we had 18/34/18 fibers allocated to sources brighter or equal to 27.2 and in good regions of the CCD. In total, we detected 15/12/13 sharp line emitters, and 0/2/0 Ly $\alpha$ emitters which show one resolved asymmetric line. The remaining spectra did not show any spectral features in the wavelength range covered by FLAMES. The fraction of confirmed spectra with both components of the [OIII] doublet detected is $67 \% / 41 \% / 18 \%$.

The extracted 1D spectra for the CORE and SUB fields are noisier than those for the FCJ, most probably because the selected reference stars for the FLAMES MEDUSA configuration were not in the astrometric system of the ICPN candidates, therefore fibers were not optimally positioned. For the SUB field, also the observing conditions were slightly worse than for the other fields, resulting in a low fraction of confirmed spectra with detected [OIII] doublet. In the CORE field we checked that the summed spectrum of all sharp line emitters also shows the $\lambda 4959 \AA$ emission in addition to the $\lambda 5007 \AA$ line. The line ratio is 3.5, but with large error because of the noise in the summed spectrum: these spectra are compatible with being all ICPNe.

Table 1 also gives the spectroscopic confirmation rates in the three fields, for candidates down to the brighter of 27.2 or $m_{\text {lim }}(5007)$ from the photometry. This rate varies strongly from field to field despite similar $m_{\text {lim }}(5007)$. The most important reason for this is different contamination of the samples by faint continuum stars, erroneously classified as ICPNe because of a shallow off-band image. Aguerri et al. (2004) investigated the contamination of the photometric samples caused by faint continuum stars, [OII] emitters at $z=0.347$, and high-z emitters, using simulations and blank field surveys. Table 1 shows the expected spectroscopic confirmation rates based on these simulations. These are in close agreement with the actual confirmation rates, showing that the photometric samples are well understood. A high confirmation rate (small 


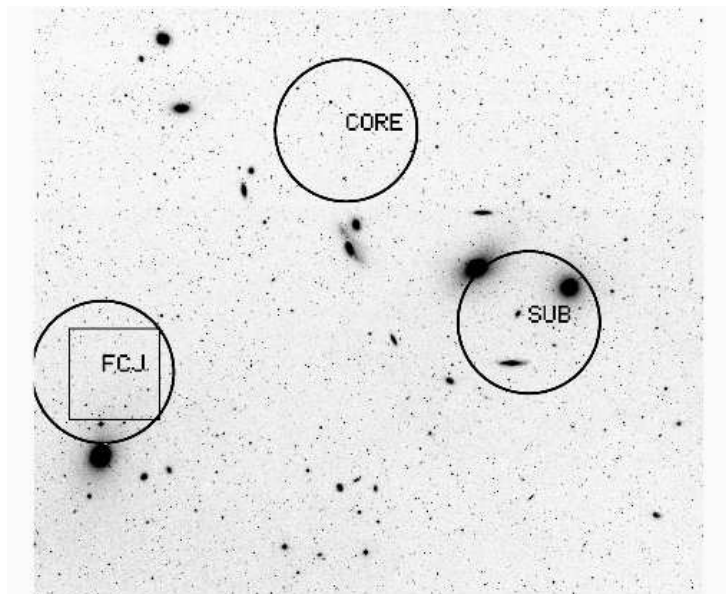

FIG. 2.- Pointings surveyed in the Virgo core region. The field shown is $2^{\circ} \times 2^{\circ}$ with M87 near FCJ and M86/M84 near SUB. The circles show the FLAMES FOVs. The square in the FCJ pointing marks the area of the imaging surveyed field.

contamination) can be achieved when the off-band image is sufficiently deep. See Aguerri et al. (2004) for further details.

\section{ICPN LINE-OF-SIGHT VELOCITY DISTRIBUTIONS}

With these data, we can now for the first time determine radial velocity distributions of ICPNe and use these to investigate the dynamical state of the Virgo cluster. Fig. 2 shows an image of the Virgo cluster core with the positions of our FLAMES pointings. The heliocentric radial velocity histograms obtained from the spectra in these fields are displayed in Fig. 3 Clearly the histograms for the three pointings are very different.

In the FCJ field, the velocity distribution of the PNe is not consistent with a single Gaussian with either the fitted velocity dispersion $\sigma_{\mathrm{FCJ}}=573 \mathrm{~km} \mathrm{~s}^{-1}$, or the more canonical Virgo $\sigma=800 \mathrm{~km} \mathrm{~s}^{-1}$, based on a $\chi^{2}$-test. Instead, it is dominated by a narrow peak, with $\bar{v}_{p}=1276 \pm 71 \mathrm{~km} \mathrm{~s}^{-1}$ and $\sigma_{p}=247 \pm 52$ $\mathrm{km} \mathrm{s}^{-1}$, which we identify with the halo of M87 below. In addition, there are 3 outliers, 2 at low velocity. All three are unusually bright, in the bright fall-off of the M87 PN luminosity function (PNLF), and they shift the FCJ luminosity function to a brighter cutoff (Arnaboldi et al. 2002). The ICL surface brightness associated with these 3 outliers, e.g. the likely true ICPNe in the FCJ field, is $\mu_{B} \simeq 30.7 \mathrm{mag} \mathrm{arcsec}^{-2}$ if we use the conversion for M31 from Ciardullo et al. (1989), $\alpha_{1, B}$. This is comparable with the surface brightness measurements of Ferguson et al. (1998) and Durrell et al. (2002) from intracluster red giants in fields further away from M87.

The M87 peak of the FCJ velocity distribution contains 12 velocities, which are described well by a Gaussian according to KS and $\chi^{2}$ tests. Their luminosity function is consistent with the PNLF in the inner $4^{\prime}$ of M87 (Ciardullo et al. $1998)$, with a KS probability $p_{K S}=0.46$. The average velocity agrees with that of M87, $v_{s y s}=1307 \mathrm{~km} \mathrm{~s}^{-1}$. The value of $\sigma_{p}$ is consistent with the stellar velocity dispersion profile extrapolated outwards from $\simeq 150^{\prime \prime}$ in Figure 5 of Romanowsky $\&$ Kochanek (2001) and falls in the range spanned by their dynamical models for the M87 stars (the center of FCJ is $15 .^{\prime} 0 \simeq 65 \mathrm{kpc}$ from M87, for an assumed M87 distance of 15 $\mathrm{Mpc})$. To infer the surface brightness corresponding to the 12 PNe in the M87 peak, we again use $\alpha_{1, B}$ for M31, because of the colour gradient observed in the outer parts of M87 (Goud-

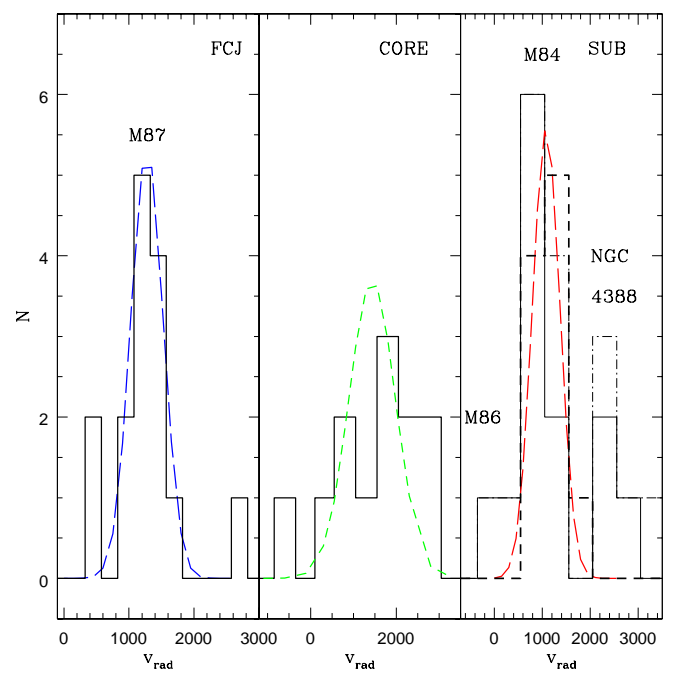

FIG. 3.- ICPN radial velocity distributions in the three pointings (FCJ, CORE, and SUB). In the FCJ panel, the blue dashed line shows a Gaussian with $\bar{v}_{\text {rad }}=1276 \mathrm{~km} \mathrm{~s}^{-1}$ and $\sigma_{\text {rad }}=247 \mathrm{~km} \mathrm{~s}^{-1}$. In the CORE panel, the green dashed line shows a Gaussian with $\bar{v}_{\text {rad }}=1436 \mathrm{~km} \mathrm{~s}^{-1}$ and $\sigma_{\text {rad }}=538 \mathrm{~km} \mathrm{~s}^{-1}$, for $\mathrm{VC}$ galaxies $\mathrm{dE}$ and dS0 within $2^{\circ}$ of M87 (from Binggeli et al. 1987). In the SUB sub, the overplotted dashed histogram shows the radial velocities from a TNG spectroscopic follow-up (Arnaboldi et al. 2003). The dashed red line shows a Gaussian with $\bar{v}_{\text {rad }}=1079 \mathrm{~km} \mathrm{~s}^{-1}$ and $\sigma_{\text {rad }}=286 \mathrm{~km} \mathrm{~s}^{-1}$ for the M84 peak. The overplotted dashed-dotted lines show the SUB-FLAMES spectra including those of HII regions, which have radial velocities in the M84 and NGC 4388 velocity ranges.

frooij et al. 1994). The resulting $\mu_{B}=29.2 \mathrm{mag} \mathrm{arcsec}^{-2}$ falls on the average M87 surface brightness profile extrapolated from $\simeq 400^{\prime \prime} . \quad \mu_{B}$ and $\sigma_{p}$ are thus also consistent with the extrapolated dynamical model of Romanowsky \& Kochanek (2001) for the M87 stars, in the distribution of dark matter inferred by them, which is also similar to that determined by Matsushita et al. (2002). By contrast, the available data and dynamical models show an approximately flat dispersion profile for the globular clusters in $\mathrm{M} 87$, at $\sigma_{\mathrm{GC}} \simeq 350 \mathrm{~km} \mathrm{~s}^{-1}$, corresponding to a shallower radial distribution. The main result from our measurement of $\sigma_{p}$ is that M87 has a stellar halo in approximate dynamical equilibrium out to at least $65 \mathrm{kpc}$.

In the CORE field, the distribution of ICPN line-of-sight (LOS) velocities is clearly broader than in the FCJ field and consistent with a Gaussian $\left(p_{K S}=0.9\right)$. It has $\bar{v}_{C}=1491 \pm 290$ $\mathrm{km} \mathrm{s}^{-1}$ and $\sigma_{C}=1000 \pm 210 \mathrm{~km} \mathrm{~s}^{-1}$; the median is $1791 \mathrm{~km}$ $\mathrm{s}^{-1}$. The CORE field is in a region of Virgo devoid of bright galaxies, but contains 7 dwarfs, and 3 low luminosity E/S near its $\mathrm{S} / \mathrm{W}$ borders. None of the confirmed ICPNe lies within a circle of three times half the major axis diameter of any of these galaxies, and there are no correlations of their velocities with the velocities of the nearest galaxies where these are known. Thus in this field there is a clear IC stellar component.

The mean velocity of the ICPN in this field is similar to that of 25 Virgo $\mathrm{dE}$ and dS0 within $2^{\circ}$ of M87, $\left.<v_{\mathrm{dE}, \mathrm{M} 87}\right\rangle=$ $1436 \pm 108 \mathrm{~km} \mathrm{~s}^{-1}$ (Binggeli et al. 1987), and with that of $93 \mathrm{dE}$ and dS0 Virgo members, $\left\langle v_{\mathrm{dE} \text {, Virgo }}>=1139 \pm 67 \mathrm{~km}\right.$ $\mathrm{s}^{-1}$ (Binggeli et al. 1993). However, the velocity dispersion of these galaxies is smaller, $\sigma_{\mathrm{dE}, \mathrm{M} 87}=538 \pm 77 \mathrm{~km} \mathrm{~s}^{-1}$ and $\sigma_{\mathrm{dE}, \text { Virgo }}=649 \pm 48 \mathrm{~km} \mathrm{~s}^{-1}$.

The inferred luminosity from the ICPNe in the CORE field is $1.8 \times 10^{9} L_{B, \odot}$ (Aguerri et al. 2004). This is about 
three times the luminosity of all dwarf galaxies in this field, $5.3 \times 10^{8} L_{B, \odot}$, but an order of magnitude less than the luminosities of the three low-luminosity E/S galaxies near the field borders. Using the results of Nulsen \& Böhringer (1995) and Matsushita et al. (2002), we estimate the mass of the M87 subcluster inside $310 \mathrm{kpc}$ (the projected distance $D$ of the CORE field from M87) as $4.2 \times 10^{13} M_{\odot}$, and compute a tidal parameter $T$ for all these galaxies as the ratio of the mean mass density within $D$ to the mean density of the galaxy. We find $T=0.01-0.06$, independent of galaxy luminosity. Since $T \sim D^{-2}$, any of these galaxies whose orbit now comes closer to M87 than $\sim 60 \mathrm{kpc}$ would be subject to severe tidal mass loss. Thus the ICPN population in the CORE field could be debris from the tidal disruption of small galaxies on nearby orbits in the M87 halo. The relatively small number of ICPNe in the FCJ field at $D=65 \mathrm{kpc}$ could then mean that most of the tidally disrupted galaxies did not orbit as deep into M87.

In the SUB field the velocity distribution from FLAMES spectra is again different from CORE and FCJ. The KS test gives low probabilities, $p_{K S}=0.13$ and $p_{K S}=0.03$, that the SUB histogram could be drawn from the velocity histogram in the CORE field or the Gaussian fitted to this, respectively. Instead, the SUB histogram of LOS velocities shows substructures that are highly correlated with the systemic velocities of M86, M84 and NGC 4388. The association with the three galaxies is strengthened when we plot the LOS velocities of 4 HII regions (see Gerhard et al. 2002) detected with FLAMES in this pointing. The highest peak in the distribution coincides with M84, and even more so when we add the LOS velocities obtained previously at the TNG (Arnaboldi et al. 2003). The $10 \mathrm{TNG}$ velocities ( 2 velocities were measured in addition to the Arnaboldi et al. (2003) sample) give $\bar{v}_{\mathrm{M} 84}=1079 \pm 103 \mathrm{~km} \mathrm{~s}^{-1}$ and $\sigma_{\mathrm{M} 84}=325 \pm 75 \mathrm{~km}$ $\mathrm{s}^{-1}$ within a square of $4 R_{e} \times 4 R_{e}$ of the M84 center. The 8 FLAMES velocities in the M84 subpeak give $\bar{v}_{\mathrm{M} 84}=891 \pm 74$ $\mathrm{km} \mathrm{s}^{-1}$ and $\sigma_{\mathrm{M} 84}=208 \pm 54 \mathrm{~km} \mathrm{~s}^{-1}$, going out to larger radii. Note that this includes three over-luminous PNe not attributed to M84 previously. The combined sample of 18 velocities gives $\bar{v}_{\mathrm{M} 84}=995 \pm 69 \mathrm{~km} \mathrm{~s}^{-1}$ and $\sigma_{\mathrm{M} 84}=293 \pm 50 \mathrm{~km} \mathrm{~s}^{-1}$. Most likely, all these PNe belong to a very extended envelope around M84 (see the deep image in Arnaboldi et al. 1996). It is possible that the somewhat low velocity with respect to M84 may be a sign of tidal stripping by M86, or of a recent merger with a smaller galaxy. In the latter case, the overluminous PNe might be due to a younger or a more metal-poor population (Dopita et al. 1992).

\section{CONCLUSIONS}

We have presented the first measurements of the velocity distribution of ICPNe in three fields of the Virgo cluster. Overall, these velocity measurements confirm the view that Virgo is a highly non-uniform and unrelaxed galaxy cluster, consisting of several subunits that have not yet have had time to come to equilibrium in a common gravitational potential.

A well-mixed IC stellar population is seen clearly only in the CORE field, in the outer parts of the M87 subcluster. Here the velocity distribution is consistent with a single cluster Gaussian, and the ICPNe might well have their origin in the tidal effects of the halo of this subcluster on its galaxy population. In the SUB field near M84 and M86, the ICPNe do not appear virialized; their velocities are highly correlated with those of the large galaxies in the field. In fact, there are regions in Virgo where no ICPNe are found (the LPC field of Aguerri et al. 2004).

The measurements have also shown that M87 has a very extended envelope in approximate dynamical equilibrium, reaching out to at least $65 \mathrm{kpc}$. The cluster ICPN population in our FCJ field has density comparable with that in other fields further out, indicating a shallow ICL density distribution.

M.A. would like to thank L. Pasquini and F. Primas for their help during the VLT-FLAMES observing block preparation and service observing, and $\mathrm{C}$. Cacciari, E. Pancino and E. Rossetti for their help with the GIRAFFE pipeline. We thank B. Binggeli and M. Capaccioli for useful discussions, B. Binggeli for providing his Virgo cluster galaxy catalogue with the $\mathrm{dE} \& \mathrm{dS} 0$ radial velocity data, and an anonymous referee for constructive comments. We acknowledge financial support by INAF - Projects of National Interests (P.I. MA), SNF grant 200020-101766, and Spanish DGES grant AYA20013939. NRN is supported by a MC fellowship within the EC Fifth Framework Program. This research has made use of the NASA/IPAC Extragalactic Database (NED).

\section{REFERENCES}

Aguerri, J.A.L., Gerhard, O.E., Arnaboldi, M., Napolitano, N.R., CastroRodriguez, N., \& Freeman, K.C. 2004, AJ, submitted

Arnaboldi, M. et al. 2003, AJ, 125, 514

Arnaboldi, M. et al. 2002, AJ, 123, 760

Arnaboldi, M. et al. 1996, ApJ, 472, 145

Binggeli, B., Popescu, C. C., \& Tammann, G. A., 1993, A\&A, 98, 275

Binggeli, B., Tammann, G.A., \& Sandage, A. 1987, AJ, 94, 251

Castro-Rodriguez, N., Aguerri, J.A.L., Arnaboldi, M., Gerhard, O.E., Freeman, K.C., Napolitano, N.R., \& Capaccioli, M. 2003, A\&A, 405, 803

Ciardullo, R., Jacoby, G. H., Feldmeier, J. J., \& Bartlett, R. E. 1998, ApJ, 492, 62

Ciardullo, R., Jacoby, G. H., \& Ford, H. C. 1989, ApJ, 344, 715

Durrell, P.R., Ciardullo, R. Feldmeier, J.J., Jacoby, G.H. \& Sigurdsson, S. 2002, ApJ, 570, 119

Dopita, M. A., Jacoby, G. H., \& Vassiliadis, E. 1992, ApJ, 389, 27

Feldmeier, J.J., Ciardullo, R., Jacoby, G.H. \& Durrell, P.R. 2003a, ApJS, 145, 65

Feldmeier, J.J., Ciardullo, R., Jacoby, G.H. \& Durrell, P.R. 2003b, in IAU Symposium 217, Recycling Intergalactic and Interstellar Matter, in press Feldmeier, J.J., Ciardullo, R. \& Jacoby, G.H. 1998, ApJ, 503, 109

Ferguson, H.C., Tanvir, N.R., \& von Hippel, T. 1998, Nature, 391, 461

Freeman, K.C. et al. 2000, in ASP Conf. Ser. 197: Dynamics of Galaxies: from Early Universe to the Present, 389
Gal-Yam, A.H. et al. 2000, ApJ, 536, 561

Gerhard, O., Arnaboldi, M., Freeman, K.C., Okamura, S. 2002, ApJ, 580, L121

Gonzalez, A. H., Zabludoff, A. I., Zaritsky, D., \& Dalcanton, J. J. 2000, ApJ, 536,561

Goudfrooij, P., Hansen, L., Jörgensen, H.E. \& Norgaard-Nielsen, H.U. 1994, A\&AS, 105, 341

Kudritzki, R.-P, et al. 2000, ApJ, 536, 19

Matsushita, K., Belsole, E., Finoguenov, A., Böhringer, H, 2002, A\&A, 386, 77

Murante, G. et al. 2004, ApJ, 607, L83

Napolitano, N.R. et al. 2003, ApJ, 594, 172

Nulsen, P. E. J., \& Bohringer, H., 1995, MNRAS, 274, 1093

Okamura, S. et al. 2002, PASJ, 54, 883

Romanowsky, A. J., \& Kochanek, C. S. 2001, ApJ, 553, 722

Sommer-Larsen, J., Romeo, A.D. \& Portinari, L. 2004, MNRAS, submitted (astro-ph/0403282,

Theuns, T. \& Warren, S.J. 1997, MNRAS, 284, L11

Willman, B. et al. 2004, MNRAS, submitted (astro-ph/0405094) 